\title{
MIN-MAX SOLUTIONS FOR PARAMETRIC CONTINUOUS STATIC GAME UNDER ROUGHNESS (PARAMETERS IN THE COST FUNCTION AND FEASIBLE REGION IS A ROUGH SET)
}

\author{
Yousria A. Aboelnaga \\ Higher Technological Institute, \\ Tenth of Ramadan City, 44629, Egypt \\ yousria_naga@yahoo.com \\ Mai F. Zidan \\ Faculty of Engineering, Tanta University, \\ Al-Geish St., Tanta, 31512, Egypt \\ mai.fathi@f-eng.tanta.edu.eg
}

\begin{abstract}
Any simple perturbation in a part of the game whether in the cost function and/or conditions is a big problem because it will require a game re-solution to obtain the perturbed optimal solution. This is a waste of time because there are methods required several steps to obtain the optimal solution, then at the end we may find that there is no solution. Therefore, it was necessary to find a method to ensure that the game optimal solution exists in the case of a change in the game data. This is the aim of this paper. We first provided a continuous static game rough treatment with Min-Max solutions, then a parametric study for the processing game and called a parametric rough continuous static game (PRCSG). In a Parametric study, a solution approach is provided based on the parameter existence in the cost function that reflects the perturbation that may occur to it to determine the parameter range in which the optimal solution point keeps in the surely region that is called the stability set of the $1^{s t}$ kind. Also the sets of possible upper and lower stability to which the optimal solution belongs are characterized. Finally, numerical examples are given to clarify the solution algorithm
\end{abstract}

Keywords: Continuous static game, Rough programming, Non-linear programming, Rough set theory, Parametric linear programming, Parametric non-linear programming.

\section{Introduction}

Rough programming is introduced in $[7,8,13,19]$. It is classified into three classes based on the roughness detected [13]; $1^{\text {st }}$ class: the roughness exists in a feasible set with crisp objective function; $2^{\text {nd }}$ class: the roughness exists in the objective function with a crisp feasible set; $3^{\text {rd }}$ class: the roughness exists in both feasible set and objective function. Therefore, the nonlinear programming problem $[2,3,11]$ with a rough representation is called a rough nonlinear programming problem (RNPP) and can be defined by roughness, which may be in constraints and/or objective functions. The RNPP has two solution sets: possibly and surely feasible optimal solution sets in the $1^{\text {st }}$ class. Parametric optimization [6] is a helpful universal tool that has recently got many applications in process engineering systems $[1,7,14,20]$. Given a constrained optimization problem, including a set of variables and bounded parameters, it provides the optimal value of the variables as an explicit function of the parameters without completely enumerating the entire space of the parameters. This means that if the value of the parameter changes within the given bounds, the optimal solution can 
be obtained by a simple evaluation of the explicit function without having to resolve an optimization problem. This is a powerful result that has been successfully applied in multiobjective optimization $[10,15]$, stochastic optimization $[4,16]$, flexibility analysis, hybrid, and robust model-based control $[5,17]$. This paper investigates the analysis of basic concepts in a parametric rough continuous static game when parameters are in the cost functions and roughness is in the constraints which are not discussed before. A survey of rough optimality, rough set theory (RST), and rough functions is necessary for parametric studying of RNPP.

This article is structured as follows: Section 2 introduces Min-Max solutions for the rough continuous static game of $1^{\text {st }}$ class with an illustrative example to explain the solution steps. In Section 3, the Rough Continuous Static game parametric study when roughness exists in the constraints and parameters in the objective functions is discussed with a numerical example to clear up the presented concepts. Section 4 covers the conclusion and future studies.

\section{2. $1^{\text {st }}$ class of Rough Continuous Static game (RCSG)}

The continuous static game (CSG) $[9,17,18]$ is defined as an optimization problem where each player in the game controls a specified subset of the system parameters and seeks to minimize his own cost criterion subject to specified constraints. In these games control notation is used and each player $i=1, \ldots, r$ selects his control vector $u^{i} \in E^{s}$ seeking to minimize a scalar valued criterion

$$
G_{i}(x, u)
$$

subject to $n$ equality constrains

$$
g(x, u)=0
$$

where $x \in E^{s}$ is the state and

$$
u=\left(u^{1}, \ldots, u^{r}\right) \in E^{s}, \quad s=s_{1}+\ldots+s_{r}
$$

is the composite control. The composite control is required to be an element of a regular control constraint set $\Omega \subseteq E^{s}$ of the form:

$$
\Omega=\left\{u \in E^{s} \mid h(x, u) \geq 0\right\},
$$

where $x=\zeta(u)$ is the solution of $(2.2)$ given $u$. The functions

$$
G_{i}(x, u): E^{n} * E^{s} \rightarrow E^{1}, \quad g(x, u): E^{n} * E^{s} \rightarrow E^{n}, \quad h(x, u): E^{n} \rightarrow E^{s} \rightarrow E^{q}
$$

are assumed to be $e^{1}$, with

$$
\left|\frac{\partial g(x, u)}{\partial x}\right| \neq 0
$$

in a ball around a solution point $(x, u)$. The above problem can be written as:

$$
\begin{gathered}
\min G_{i}(x, u), \\
\text { S.T. } \\
M=\left\{x \in E^{n}, u \in E^{s} \mid g(x, u)=0, h(x, u) \geq 0\right\},
\end{gathered}
$$

where $G_{i}$ is called the cost function for each player $i=1, \ldots, r$, and $M$ is called the feasible set of the problem. 
Definition 1. Let $A(U, R)$ be an approximation space, $U$ be the universe and $R$ be an equivalence relation on $U$. Let $M$ be a subset of $U$, i.e. $M \subseteq U$. Then, the $1^{\text {st }}$ class of RCSG can be defined as follows:

$$
\begin{gathered}
\min G_{i}(x, u), \\
S . T . \\
M_{*} \subseteq M \subseteq M^{*},
\end{gathered}
$$

where $M_{*}, M^{*}$ are lower and upper approximation of the feasible set, respectively. The solution sets of the $1^{\text {st }}$ class of RCSG are called a surely optimal solution set and a possibly optimal solution set. To find such solutions, first solve the following problem:

$$
\begin{gathered}
\min G_{i}(x, u), \\
S . T . \\
m \in M^{*} .
\end{gathered}
$$

The optimal solution set of the above game is

$$
O=\left\{\tilde{m} \in M^{*} \mid G_{i}(x, \tilde{m})=\min _{m \in M^{*}} G_{i}(x, u)\right\} .
$$

The optimal solution value of the cost function on upper approximation set is

$$
G^{*}=\min _{m \in M^{*}} G_{i}(x, u) .
$$

There are now two possibilities: one providing surely optimal solution, possibly optimal solution and another one leads us to resolve the game on the lower approximation as follows:

- If $O_{1}=O \cap M_{*} \neq \emptyset$, then a solution set $O_{1}$ is called a surely optimal solution set and $O \sim O_{1}$ is possibly optimal solution set.

- If $O_{1}=O \cap M_{*}=\emptyset$, then $O \subseteq M_{B N}$ (boundary approximation of feasible region). In this case, the game does not have surely optimal solution set. Therefore, it will be resolved on the lower approximation to obtain $\mathrm{O}_{2}$, which is an optimal solution set on the lower approximation.

Where, the game on the lower approximation is

$$
\begin{gathered}
\min G_{i}(x, u), \\
\text { S.T. } \\
M_{*} \subseteq M,
\end{gathered}
$$

and the optimal solution value of the cost function on lower approximation set is

$$
G_{*}=\min _{m \in M_{*}} G_{i}(x, u)
$$

Definition 2. If $O_{1} \neq \emptyset$, then $O_{1}$ contains all surely optimal solutions, hence it is called the surely optimal set $O_{1} \subseteq M$.

Definition 3. $O \sim O_{1}$ contains possibly optimal solutions, hence it is called the possibly optimal set because $O \sim O_{1} \subseteq M_{B N}$. 


\subsection{Min-Max solutions for the $1^{\text {st }}$ class of RCSG}

For this game player $i$ chooses his control under the assumption that all players have formed a coalition to maximize his cost. In other words, all players expect one cooperate against the remaining player $i$.

Definition 4. A point $w_{*}=\left(u_{*_{i}}, v_{*}\right)$ is a completely regular lower point if and only if $w_{*}$ is a regular point of $\Omega$ and $u_{*_{i}}$ is a regular point of $U_{*_{i}}$ for each $i=1, \ldots, r$. Here $u_{*_{i}}$ is a lower control vector for each player $i=1, \ldots, r$ and $v_{*}$ is the composite lower control of the remaining players, $h_{*}(\cdot)$ defines lower inequality constraints and

$$
U_{*_{i}}=\left\{u_{*_{i}} \in E^{s} \mid h_{*}\left[\zeta\left(u_{*_{i}}, v_{*}\right), u_{*_{i}}, v_{*}\right] \geq 0\right\} .
$$

Definition 5. A point $\tilde{u}_{*} \in \Omega$ is a rough min-max lower point for player $i$ if and only if

$$
G_{i}\left[\zeta\left(\tilde{u}_{*_{i}}, v_{*}\right), \tilde{u}_{*_{i}}, v_{*}\right] \leq G_{i}\left[\zeta\left(\tilde{u}_{*}\right), \tilde{u}_{*}\right] \leq G_{i}\left[\zeta\left(u_{*_{i}}, \tilde{v}_{*}\right), u_{*_{i}}, \tilde{v}_{*}\right]
$$

for all $u_{*_{i}} \in U_{*_{i}}$ where $\tilde{u}_{*}=\left(\tilde{u}_{*_{i}}, \tilde{v}_{*}\right), U_{*_{i}}$ is defined by (2.5) and $x_{*}=\zeta\left(u_{*}\right)$ is the solution to $M_{*}\left(x_{*}, \tilde{u}_{*}\right)=0$. For a local rough min-max lower point replace $U_{*_{i}}$ by $B_{i} \cap U_{*}$ for some ball $B_{i} \subset E^{s}$ centered at $\tilde{u}_{*}$.

Lemma 1. If $\tilde{u}_{*}=\left(u_{i_{*}}, v_{*}\right) \in \Omega$ is a local min-max lower point for player $i$ for the game (2.3)(2.4), and if $x_{*}=\zeta\left(u_{*}\right)$ is the solution to $M_{*}\left(x_{*}, \tilde{u}_{*}\right)=0$, then there exists a vector $\gamma_{*_{i}} \in E^{n}$ defined by

$$
\frac{\partial J_{*_{i}}\left[x_{*}, \tilde{u}_{*}, \gamma_{*_{i}}\right]}{\partial x_{*}}=0, \quad \frac{\partial J_{*_{i}}\left[x_{*}, \tilde{u}_{*}, \gamma_{*_{i}}\right]}{\partial u_{*_{i}}} e^{i}=0
$$

for all $e^{i} \in T_{*_{i}}$ and

$$
\frac{\partial J_{*_{i}}\left[x_{*}, \tilde{u}_{*}, \gamma_{*_{i}}\right]}{\partial v_{*}} e^{v}=0
$$

for all $e^{v} \in T_{*_{v}}$, where

$$
J_{*_{i}}\left[x_{*}, \tilde{u}_{*}, \gamma_{*_{i}}\right]=G_{*_{i}}\left(x_{*}, u_{*}\right)-\gamma_{*_{i}}^{T} g_{*}\left(x_{*}, u_{*}\right),
$$

and the tangent cones $T_{*_{i}}$ and $T_{*_{v}}$ are given by

$$
\begin{gathered}
T_{*_{i}}=\left\{e^{i} \in E^{s_{i}} \mid\left[\frac{\partial h_{*}}{\partial u_{*_{i}}}-\frac{\partial h_{*}}{\partial x_{*}}\left[\frac{\partial g_{*}}{\partial x_{*}}\right]^{-1} \frac{\partial g_{*}}{\partial u_{*_{i}}}\right] e^{i}\right\} \geq 0, \\
T_{*_{v}}=\left\{e^{i} \in E^{s-s_{i}} \mid\left[\frac{\partial h_{*}}{\partial v_{*}}-\frac{\partial h_{*}}{\partial x_{*}}\left[\frac{\partial g_{*}}{\partial x_{*}}\right]^{-1} \frac{\partial g_{*}}{\partial v_{*}}\right] e^{v}\right\} \geq 0,
\end{gathered}
$$

where $h_{*}$ denotes the active inequality constraints at $u_{*}=\left(u_{*_{i}}, v_{*}\right)$.

Proposition 1. If $\tilde{u}_{*}=\left(u_{*}, v_{*}\right) \in \Omega$ is a completely regular rough min-max lower point for the player $i$ and $x_{*}=\zeta\left(\tilde{u}_{*}\right)$ is the solution to $M_{*}\left(x_{*}, \tilde{u}_{*}\right)=0$, then there exist vectors $\lambda_{*_{i}} \in E^{n}$, 
$\bar{\lambda}_{*_{i}} \in E^{n}, \mu_{*_{i}} \in E^{q}$ and $\bar{\mu}_{*_{i}} \in E^{q}$ such that:

$$
\begin{gathered}
\frac{\partial L_{*_{i}}\left[x_{*}, \tilde{u}_{*}, \lambda_{*_{i}}, \mu_{*_{i}}\right]}{\partial x_{*}}=0, \\
\frac{\partial L_{*_{i}}\left[x_{*}, \tilde{u}_{*}, \bar{\lambda}_{*_{i}}, \bar{\mu}_{*_{i}}\right]}{\partial x_{*}}=0, \\
\frac{\partial L_{*_{i}}\left[x_{*}, \tilde{u}_{*}, \lambda_{*_{i}}, \mu_{*_{i}}\right]}{\partial u_{*}}=0, \\
\frac{\partial L_{*_{i}}\left[x_{*}, \tilde{u}_{*}, \bar{\lambda}_{*_{i}}, \bar{\mu}_{*_{i}}\right]}{\partial v_{*}}=0, \\
M_{*}\left(x_{*}, \tilde{u}_{*}\right)=0 \\
\mu_{*_{i}}^{T} h_{*}\left(\tilde{x}, \tilde{u}_{*}\right)=0, \\
\bar{\mu}_{*_{i}}^{T} h_{*}\left(x_{*}, \tilde{u}_{*}\right)=0 \\
h_{*}\left(\tilde{x}, \tilde{u}_{*}\right) \geq 0 \\
\mu_{*_{i}} \geq 0 \\
\bar{\mu}_{*_{i}} \leq 0
\end{gathered}
$$

where

$$
\begin{aligned}
& L_{*_{i}}\left[x_{*}, \tilde{u}_{*}, \lambda_{*_{i}}, \mu_{*_{i}}\right]=G_{i}\left(x_{*}, u_{*}\right)-\lambda_{*_{i}} M_{*}\left(x_{*}, u_{*_{i}}\right)-\mu_{*_{i}} h_{*}\left(x_{*}, u_{*_{i}}\right), \\
& L_{*_{i}}\left[x_{*}, \tilde{u}_{*}, \bar{\lambda}_{*_{i}}, \bar{\mu}_{*_{i}}\right]=G_{i}\left(x_{*}, u_{*}\right)-\bar{\lambda}_{*_{i}} M_{*}\left(x_{*}, u_{*_{i}}\right)-\bar{\mu}_{*_{i}} h_{*}\left(x_{*}, u_{*_{i}}\right) .
\end{aligned}
$$

P r o o f. For player $i$ consider the cones

$$
\begin{gathered}
K_{*_{i}}=\left\{y_{*_{i}} \in E^{s_{i}} \mid y_{*_{i}}{ }^{T}=\mu_{*_{i}}{ }^{T}\left[\frac{\partial h_{*}}{\partial u_{*_{i}}}-\frac{\partial h_{*}}{\partial x_{*}}\left[\frac{\partial g_{*}}{\partial x_{*}}\right]^{-1} \frac{\partial g_{*}}{\partial u_{*_{i}}}\right], \quad \mu_{*_{i}}{ }^{T} h_{*}=0, \mu_{*_{i}} \geq 0\right\}, \\
K_{*_{2}}=\left\{y_{*_{2}} \in E^{s-s_{i}} \mid y_{*_{2}}{ }^{T}=\mu_{*_{i}}{ }^{T}\left[\frac{\partial h_{*}}{\partial v_{*}}-\frac{\partial h_{*}}{\partial x_{*}}\left[\frac{\partial g_{*}}{\partial x_{*}}\right]^{-1} \frac{\partial g_{*}}{\partial v_{*}}\right], \quad \mu_{*_{2}}{ }^{T} h_{*}=0, \mu_{*_{2}} \geq 0\right\},
\end{gathered}
$$

and their polars, respectively,

$$
\begin{aligned}
& K^{P}{ }_{*_{i}}=\left\{Z_{*_{i}} \in E^{s_{i}} \mid y_{*_{i}}{ }^{T} Z_{*_{i}} \geq 0 \quad \forall y_{*_{i}} \in K_{*_{i}}\right\}, \\
& K^{P}{ }_{*_{2} i}=\left\{Z_{*_{2}} \in E^{s-s_{i}} \mid y_{*_{2}}^{T} Z_{*_{2}} \geq 0 \quad \forall y_{*_{2}} \in K_{*_{2}}\right\} \text {. }
\end{aligned}
$$

Since $u_{*_{i}}$ is a regular point of $U_{*_{i}}$ the tangent cone $T_{i_{*}}$ to $U_{*_{i}}$ is given by

$$
T_{*_{i}}=K_{*_{i}}^{P_{i}}, \quad T_{*_{v}}=K_{*_{2}}^{P_{2}} .
$$

From this result and Lemma 1 we have

$$
\begin{aligned}
& \frac{\partial J_{*_{i}}\left[x_{*}, \tilde{u}_{*}, \gamma_{*_{i}}\right]}{\partial u_{*_{i}}} \in K_{*_{i}}, \\
& \frac{\partial J_{*_{i}}\left[x_{*}, \tilde{u}_{*}, \gamma_{*_{i}}\right]}{\partial v_{*}} \in K_{*_{2}},
\end{aligned}
$$

where $J_{*_{i}}$ is defined by (2.6) and $\gamma_{*_{i}}$ is defined by

$$
\frac{\partial J_{*_{i}}\left[x_{*}, \tilde{u}_{*}, \gamma_{*_{i}}\right]}{\partial x_{*}}=0 .
$$


Define

$$
\begin{gathered}
\lambda_{*_{i}}{ }^{T}=\gamma_{*_{i}}{ }^{T}-\mu_{*_{i}}^{T} \frac{\partial h}{\partial x_{*}}\left[\frac{\partial g_{*}}{\partial x_{*}}\right]^{-1}, \\
\tilde{\lambda}_{*_{i}}^{T}=\gamma_{*_{i}}^{T}-\mu_{*_{2}}^{T} \frac{\partial h}{\partial x_{*}}\left[\frac{\partial g_{*}}{\partial x_{*}}\right]^{-1},
\end{gathered}
$$

with $L_{*_{i}}$ defined by (2.15) and choosing $\mu_{*_{i}}-\tilde{\mu}_{*_{i}}=0$, equation (2.7) follows from (2.18) by substituting for $\gamma_{*_{i}}$ from (2.19). Similarly (2.9), (2.11) and (2.13) follow from (2.16), (2.19) and the definition of $k_{*_{i}}$. Choosing $\tilde{\mu}_{*_{i}}=-\tilde{\mu}_{*_{2}} \leq 0$ and substituting for $\gamma_{*_{i}}$ from (2.20) we see that the equation (2.8) follows from (2.18). Similarly (2.10), (2.12) and (2.14) follow from (2.17) and from the definition of $k_{*_{2}}$.

Definition 6. A point $w^{*}=\left(u_{i}^{*}, v^{*}\right)$ is a completely regular upper point if and only if $w^{*}$ is a regular point of $\Omega$ and $u_{i}^{*}$ is a regular point of $U_{i}^{*}$ for each $i=1, \ldots, r$. Here $u_{i}^{*}$ is a lower control vector for each player $i=1, \ldots, r$ and $v^{*}$ is the composite lower control of the remaining players, $h^{*}(\cdot)$ defines lower inequality constraints and

$$
U_{i}^{*}=\left\{u_{i}^{*} \in E^{s} \mid h^{*}\left[\zeta\left(u_{i}^{*}, v^{*}\right), u_{i}^{*}, v^{*}\right] \geq 0\right\}
$$

Definition 7. A point $\tilde{u}^{*} \in \Omega$ is a rough min-max upper point for player $i$ if and only if

$$
G_{i}\left[\zeta\left(\tilde{u}_{i}^{*}, v^{*}\right), \tilde{u}_{i}^{*}, v^{*}\right] \leq G_{i}\left[\zeta\left(\tilde{u}^{*}\right), \tilde{u}^{*}\right] \leq G_{i}\left[\zeta\left(u_{*_{i}}, \tilde{v}_{*}\right), u_{*_{i}}, \tilde{v}_{*}\right]
$$

For all $u_{i}^{*} \in U_{i}^{*}$ where $\tilde{u}^{*}=\left(\tilde{u}_{i}^{*}, \tilde{v}^{*}\right), U_{i}^{*}$ is defined by (2.21) and $x^{*}=\zeta\left(u^{*}\right)$ is the solution to $M^{*}\left(x^{*}, \tilde{u}^{*}\right)=0$. For a local rough min-max lower point replace $U_{i}^{*}$ by $B_{i} \cap U^{*}$ for some ball $B_{i} \subset E^{s}$ centered at $\tilde{u}^{*}$.

Proposition 2. If $\tilde{u}^{*}=\left(u^{*}, v^{*}\right) \in \Omega$ is a completely regular rough min-max upper point for the player $i$ and $x^{*}=\zeta\left(\tilde{u}^{*}\right)$ is the solution to $M^{*}\left(x^{*}, \tilde{u}^{*}\right)=0$, then there exist vectors $\lambda_{i}^{*} \in E^{n}$, $\bar{\lambda}_{i}^{*} \in E^{n}, \mu_{i}^{*} \in E^{q}$ and a vector $\bar{\mu}_{i}^{*} \in E^{q}$ such that:

$$
\begin{gathered}
\frac{\partial L_{i}^{*}\left[x^{*}, \tilde{u}^{*}, \lambda_{i}^{*}, \mu_{i}^{*}\right]}{\partial x^{*}}=0, \quad \frac{\partial L_{i}^{*}\left[x^{*}, \tilde{u}^{*}, \bar{\lambda}_{i}^{*}, \bar{\mu}_{i}^{*}\right]}{\partial x^{*}}=0, \quad \frac{\partial L_{i}^{*}\left[x^{*}, \tilde{u}^{*}, \lambda_{i}^{*}, \mu_{i}^{*}\right]}{\partial u^{*}}=0, \quad \frac{\partial L_{i}^{*}\left[x^{*}, \tilde{u}^{*}, \bar{\lambda}_{i}^{*}, \bar{\mu}_{i}^{*}\right]}{\partial v^{*}}=0, \\
M^{*}\left(x^{*}, \tilde{u}^{*}\right)=0, \quad \mu_{i}^{*^{T}} h^{*}\left(\tilde{x}, \tilde{u}^{*}\right)=0, \quad \bar{\mu}_{i}^{* T} h^{*}\left(\tilde{x}, \tilde{u}^{*}\right)=0, \quad h^{*}\left(\tilde{x}, \tilde{u}^{*}\right) \geq 0, \quad \mu_{i}^{*} \geq 0, \quad \bar{\mu}_{i}^{*} \leq 0,
\end{gathered}
$$

where

$$
\begin{aligned}
& L_{i}^{*}\left[x^{*}, \tilde{u}^{*}, \lambda_{i}^{*}, \mu_{i}^{*}\right]=G_{i}\left(x^{*}, u^{*}, v^{*}\right)-\lambda_{i}^{*} M^{*}\left(x^{*}, u^{*}, v^{*}\right)-\mu_{i}^{*} h^{*}\left(x^{*}, u^{*}, v^{*}\right), \\
& L_{i}^{*}\left[x^{*}, \tilde{u}^{*}, \lambda_{i}^{*}, \mu_{i}^{*}\right]=G_{i}\left(x^{*}, u^{*}, v^{*}\right)-\bar{\lambda}_{i}^{*} M^{*}\left(x^{*}, u^{*}, v^{*}\right)-\bar{\mu}_{i}^{*} h^{*}\left(x^{*}, u^{*}, v^{*}\right) .
\end{aligned}
$$

$\mathrm{P} r$ o o $\mathrm{f}$ is similar to proof of Proposion 1.

The numerical example below will explain how we can find the min-max optimal solution point for the $1^{\text {st }}$ class RCSG.

Example 1. Two firms sell substitutable products and seek to maximize their profits through advertising. The steady-state profits of firms 1 and 2 are taken respectively, as

$$
\begin{gathered}
H_{1}(\cdot)=5 x-u-v, \\
H_{2}(\cdot)=3 x-v-2 u .
\end{gathered}
$$


The equilibrium (steady-state) fraction of the market $x$ that firms receive is given by

$$
-7 x+u-4 x v=0 \subseteq M(\cdot) \subseteq-2 x+v-2 x u=0,
$$

where $u$ and $v$ are control vectors of firm 1 and firm 2 respectively.

Solution 1. Two firms seek to maximize $H_{1}(\cdot)$ and $H_{2}(\cdot)$. Thus, firms seek to minimize

$$
\begin{gathered}
G_{1}(\cdot)=-5 x+u+v, \\
G_{2}(\cdot)=-3 x+v+2 u .
\end{gathered}
$$

Let us solve the problem (upper approximation) using Min-Max concept:

For player 1: the game (2.22), (2.23) will be the following

$$
\begin{gathered}
\min _{u^{*} \in M^{*}} G_{1}(\cdot), \\
\text { S.T. } \\
M^{*}=-2 x^{*}+v^{*}-2 x^{*} u^{*}=0 .
\end{gathered}
$$

Here we have

$$
\begin{gathered}
L_{1}^{*}=G_{1}(\cdot)-\lambda_{1}^{*} M^{*}=-5 x^{*}+u^{*}+v^{*}-\lambda_{1}^{*}\left(-2 x^{*}+v^{*}-2 x^{*} u^{*}\right), \\
\frac{\partial L_{1}^{*}}{\partial x^{*}}=-5-\lambda_{1}^{*}\left(-2-2 u^{*}\right)=0 \quad \Rightarrow \quad u^{*}=\frac{5-2 \lambda_{1}^{*}}{2 \lambda_{1}^{*}}, \\
\frac{\partial L_{1}^{*}}{\partial u^{*}}=1-\lambda_{1}^{*}\left(-2 x^{*}\right)=0 \quad \Rightarrow \quad x^{*}=-\frac{1}{2 \lambda_{1}^{*}}, \\
\frac{\partial L_{1}^{*}}{\partial v^{*}}=1-\lambda_{1}^{*}=0 \quad \Rightarrow \quad \lambda_{1}^{*}=1 \quad \Rightarrow \quad u^{*}=\frac{3}{2}, \quad x^{*}=-\frac{1}{2} .
\end{gathered}
$$

Since $M^{*}=0 \Rightarrow V^{*}=-5 / 2$. So, Rough Min-Max upper point is $\tilde{u}^{*}=(3 / 2,-5 / 2)$ and the optimal solution value of the cost function on upper approximation is $G_{1}^{*}=1.5$.

By testing this point, we see that the lower approximation is satisfied. So the solution we have got is a surely optimal solution for player 1 and there is no need for a solution at the lower approximation.

For player 2: the game (2.22), (2.24) will be the following

$$
\begin{gathered}
\min _{v^{*} \in M^{*}} G_{2}(\cdot), \\
\text { S.T. } \\
M^{*}=-2 x^{*}+v^{*}-2 x^{*} u^{*}=0 .
\end{gathered}
$$

Here we have

$$
\begin{gathered}
L_{2}^{*}=G_{2}(\cdot)-\lambda_{2}^{*} M^{*}=-3 x^{*}+v^{*}+2 u^{*}-\lambda_{2}^{*}\left(-2 x^{*}+v^{*}-2 x^{*} u^{*}\right), \\
\frac{\partial L_{2}^{*}}{\partial x^{*}}=-3-\lambda_{2}^{*}\left(-2-2 u^{*}\right)=0 \quad \Rightarrow \quad u^{*}=\frac{3-2 \lambda_{2}^{*}}{2 \lambda_{2}^{*}}, \\
\frac{\partial L_{2}^{*}}{\partial u^{*}}=2-\lambda_{2}^{*}\left(-2 x^{*}\right)=0 \quad \Rightarrow \quad x^{*}=-\frac{1}{\lambda_{2}^{*}}, \\
\frac{\partial L_{2}^{*}}{\partial v^{*}}=1-\lambda_{2}^{*}=0 \quad \Rightarrow \quad \lambda_{2}^{*}=1 \quad \Rightarrow \quad u^{*}=\frac{1}{2}, \quad x^{*}=-1 .
\end{gathered}
$$


Since $M^{*}=0 \Rightarrow V^{*}=-3$. So, Rough Min-Max upper point is $\tilde{u}^{*}=(1 / 2,-3)$ and the optimal solution value of the cost function on upper approximation is $G_{2}^{*}=1$. By testing this point, the lower approximation is not achieved. So the game for player 2 does not have a surely optimal solution and it will be resolved at the lower approximation.

Let us continue consideration (lower approximation).

For player 2: the game is the following

$$
\begin{gathered}
\min _{v_{*} \in M_{*}} G_{2}(\cdot), \\
\text { S.T. } \\
M_{*}=-7 x_{*}+u_{*}-4 x_{*} v_{*}=0 .
\end{gathered}
$$

Here we have

$$
\begin{gathered}
L_{2_{*}}=G_{2}(\cdot)-\lambda_{2_{*}} M_{*}=-3 x_{*}+v_{*}+2 u_{*}-\lambda_{2_{*}}\left(-7 x_{*}+u_{*}-4 x_{*} v_{*}\right), \\
\frac{\partial L_{2_{*}}}{\partial x_{*}}=-3-\lambda_{2_{*}}\left(-7-4 v_{*}\right)=0 \quad \Rightarrow \quad v_{*}=\frac{3-7 \lambda_{2_{*}}}{4 \lambda_{2_{*}}}, \\
\frac{\partial L_{2_{*}}}{\partial v_{*}}=1+\lambda_{2_{*}}\left(4 x_{*}\right)=0 \quad \Rightarrow \quad x^{*}=-\frac{1}{4 \lambda_{2_{*}}}, \\
\frac{\partial L_{2_{*}}}{\partial u_{*}}=2-\lambda_{2_{*}}=0 \quad \Rightarrow \quad \lambda_{2_{*}}=2 \quad \Rightarrow \quad v_{*}=-\frac{11}{8}, \quad x_{*}=-\frac{1}{8} .
\end{gathered}
$$

Since $M_{*}=0 \Rightarrow u_{*}=-3 / 16$. So, Rough Min-Max lower point is $\tilde{u}_{*}=(-3 / 16,-11 / 8)$ and the optimal solution value of the cost function on lower approximation is $G_{2_{*}}=-1.375$.

\section{Parametric Rough Continuous Static game}

A parametric study of a Rough Continuous Static game often provides a new insight into the Rough Continuous Static game. The parameter existence in Parametric Rough Continuous Static game (PRCSG) results in two cases, the first one: roughness is in the constraints and the parameter is in the cost function and the second case: the parameter is in the constraints and roughness is in the cost function. In this paper, the first case is what our study is about and the basic concepts of the parametric convex programming which are used here are established in Osman papers [5, 12] i.e. the solvability set, the stability set of the first kind.

\subsection{The $1^{\text {st }}$ case of Parametric Rough Continuous Static game (PRCSG)}

Here we consider the case when the parameters are in the cost function and roughness is in the constraints. It can be defined as:

$$
\begin{gathered}
\min G_{i}\left(u_{i}, P_{i}\right), \\
\text { S.T. } \\
m \in M,
\end{gathered}
$$

where $G_{i}$ is a cost function for each player $i=1, \ldots, r$ and $M$ is the feasible region of the game which is a rough convex set defined by $M_{*} \subseteq M \subseteq M^{*}$, and $P_{i}$ are real parameters, $i=1, \ldots, r$.

Definition 8. The solvability set of the game (3.1), (3.2) denoted by $S$ is defined by two sets $S_{*} \subseteq S \subseteq S^{*}$, where

$$
S_{*}=\left\{P_{i} \in R^{r} \mid \min _{m \in M_{*}} G_{i}\left(u_{i}, P_{i}\right) \text { exists }\right\}, \quad S^{*}=\left\{P_{i} \in R^{r} \mid \min _{m \in M^{*}} G_{i}\left(u_{i}, P_{i}\right) \text { exists }\right\}
$$


for any $P_{i} \in S$. If $\bar{m}$ is a surely optimal solution then the stability of the $1^{\text {st }}$ kind can be defined and obtained.

Definition 9. Suppose that $P_{i} \in S$ with a corresponding surely optimal solution $\bar{m} \in O_{1}\left(P_{i}\right)$ where

$$
O_{1}\left(P_{i}\right)=\left\{\bar{m} \in M_{*} \mid G_{i}\left(\bar{m}, P_{i}\right)=\min _{m \in M^{*}} G_{i}\left(u_{i}, P_{i}\right)\right\} .
$$

Then the stability set of the $1^{\text {st }}$ kind for the game (3.1), (3.2) corresponding to $\bar{m}$ denoted by $S_{1}(\bar{m})$ is defined by

$$
S_{1}(\bar{m})=\left\{P_{i} \in S \mid G_{i}\left(\bar{m}, P_{i}\right)=\min _{m \in M^{*}} G_{i}\left(u_{i}, P_{i}\right)\right\}
$$

for any $P_{i} \in S$. If $\bar{m}$ is a possible optimal solution then the stability of the $3^{\text {rd }}$ kind can be defined and obtained.

Definition 10. Suppose that $P_{i} \in S$ with a corresponding lower optimal solution $\bar{m}_{*} \in O_{2}\left(P_{i}\right)$, where

$$
O_{2}\left(P_{i}\right)=\left\{\bar{m}_{*} \in M_{*} \mid G_{i}\left(\bar{m}_{*}, P_{i}\right)=\min _{m \in M_{*}} G_{i}\left(u_{i}, P_{i}\right)\right\} .
$$

Then the possibly lower stability set for the game (3.1), (3.2) corresponding to $\bar{m}_{*}$ denoted by $S_{2_{*}}\left(\bar{m}_{*}\right)$ is defined by

$$
S_{2_{*}}\left(\bar{m}_{*}\right)=\left\{P_{i} \in S \mid G_{i}\left(\bar{m}_{*}, P_{i}\right)=\min _{m \in M_{*}} G_{i}\left(u_{i}, P_{i}\right)\right\} .
$$

Definition 11. Suppose that $P_{i} \in S$ with a corresponding upper optimal solution $\bar{m}^{*} \in O_{3}\left(P_{i}\right)$ where

$$
O_{3}\left(P_{i}\right)=\left\{\bar{m}^{*} \in M^{*} \mid G_{i}\left(\bar{m}^{*}, P_{i}\right)=\min _{m \in M^{*}} G_{i}\left(u_{i}, P_{i}\right)\right\} .
$$

Then the possibly upper stability set for the game (3.1), (3.2) corresponding to $\bar{m}^{*}$ denoted by $S_{2}^{*}\left(\bar{m}^{*}\right)$ is defined by

$$
S_{2}^{*}\left(\bar{m}^{*}\right)=\left\{P_{i} \in S \mid G_{i}\left(\bar{m}^{*}, P_{i}\right)=\min _{m \in M^{*}} G_{i}\left(u_{i}, P_{i}\right)\right\} .
$$

\subsection{Further steps}

The steps below lead us to obtain the stability set of the $1^{\text {st }}$ kind, the possibly upper and lower stability sets when the parameters are found in cost function and roughness is in the constraints:

1. Formulate the game and include all the parameters that need to be examined.

2. For player 1, begin with a certain $P_{i} \in S$ and use Proposition 2 to get the optimal solution at upper approximation.

3. If the point that is obtained from the previous step is a surely optimal solution point then formulate Proposition 2 at it to find the stability set of $1^{\text {st }}$ kind. Otherwise, for a possible optimal solution point use Propositions 1 and 2 to obtain the possibly lower and upper stability sets.

4. Repeat for each one of the remaining players. 
Example 2. Consider the game

$$
\begin{gathered}
G_{1}(\cdot)=u^{2}+v^{2}+P_{1} u-v, \\
G_{2}(\cdot)=u^{2}+v^{2}-5 u+P_{2} v, \\
\text { S.T. }
\end{gathered}
$$$$
(u+v \leq 1) \subseteq M(\cdot) \subseteq\left(u^{2}+v^{2} \leq 9, u+v \leq 5\right) .
$$

Solution 2 (Upper approximation). Using Min-Max concept we find the following results.

For player 1: Suppose $P_{1}=-7$ and rewrite the game (3.3), (3.5) to be

$$
\begin{gathered}
\min _{u^{*} \in M^{*}} G_{1}(\cdot), \\
\text { S.T. } \\
M^{*}=\left(u^{*^{2}}+v^{*^{2}} \leq 9, u^{*}+v^{*} \leq 5\right) .
\end{gathered}
$$

We have here

$$
\begin{aligned}
& L_{1}^{*}=u^{*^{2}}+v^{*^{2}}-7 u^{*}-v^{*}+\mu_{1}^{*}\left(u^{*^{2}}+v^{*^{2}}-9\right)+\mu_{2}^{*}\left(u^{*}+v^{*}-5\right)=0, \\
& \frac{\partial L_{1}^{*}}{\partial u^{*}}=2 u^{*}-7+2 u^{*} \mu_{1}^{*}+\mu_{2}^{*}=0, \quad \frac{\partial L_{1}^{*}}{\partial v^{*}}=2 v^{*}-1+2 v^{*} \mu_{1}^{*}+\mu_{2}^{*}=0, \\
& \mu_{1}^{*}\left(u^{*^{2}}+v^{*^{2}}-9\right)=0, \quad \mu_{2}^{*}\left(u^{*}+v^{*}-5\right)=0 .
\end{aligned}
$$

When $\mu_{1}^{*}>0$ and $\mu_{2}^{*}=0$, we conclude

$$
\begin{aligned}
& 2 u^{*}-7+2 u^{*} \mu_{1}^{*}=0 \quad \Rightarrow \quad u^{*}=\frac{7}{2\left(1+\mu_{1}^{*}\right)}, \\
& 2 v^{*}-1+2 v^{*} \mu_{1}^{*}=0 \quad \Rightarrow \quad v^{*}=\frac{1}{2\left(1+\mu_{1}^{*}\right)} .
\end{aligned}
$$

Since $\mu_{1}^{*}>0 \Rightarrow u^{*^{2}}+v^{*^{2}}-9=0$. Then, $\mu_{1}^{*}=0.178511 \Rightarrow\left(u^{*}, V^{*}\right)=(2.9698,0.42445)$.

When $\mu_{2}^{*}>0$ and $\mu_{1}^{*}=0$, we conclude

$$
\begin{array}{lll}
2 u^{*}-7+\mu_{2}^{*}=0 & \Rightarrow & u^{*}=\frac{7-\mu_{2}^{*}}{2}, \\
2 v^{*}-1+\mu_{2}^{*}=0 & \Rightarrow & v^{*}=\frac{1-\mu_{2}^{*}}{2} .
\end{array}
$$

Since $\mu_{2}^{*}>0 \Rightarrow u^{*}+v^{*}-5=0$. Then, $\mu_{2}^{*}=-1$. This solution is refused.

So, the upper optimal solution point $\left(u^{*}, v^{*}\right)=(2.9698,0.42445)$ is a surely optimal solution point then the stability of the $1^{\text {st }}$ kind will be obtained as follows:

$$
2(2.9698)+P_{1}+2(2.9698) \mu_{1}^{*}=0 \Rightarrow \mu_{1}^{*}=\frac{-5.94225-P_{1}}{5.94228} .
$$

Since $\mu_{1}^{*}>0 \Rightarrow-5.94225-P_{1}>0 \Rightarrow P_{1}<-5.94225$.

So the stability of the $1^{\text {st }}$ kind is:

$$
\left(P_{1}<-5.94225\right) .
$$


For player 2: Suppose $P_{2}=-4$ and rewrite the game (3.4), (3.5) to be

$$
\begin{gathered}
\min _{u^{*} \in M^{*}} G_{2}(\cdot), \\
\text { S.T. } \\
M^{*}=\left(u^{*^{2}}+v^{*^{2}} \leq 9, u^{*}+v^{*} \leq 5\right) .
\end{gathered}
$$

We have here

$$
\begin{aligned}
& L_{1}^{*}=u^{*^{2}}+v^{*^{2}}-5 u^{*}-4 v^{*}+\mu_{1}^{*}\left(u^{*^{2}}+v^{*^{2}}-9\right)+\mu_{2}^{*}\left(u^{*}+v^{*}-5\right)=0 \text {, } \\
& \frac{\partial L_{1}^{*}}{\partial u^{*}}=2 u^{*}-5+2 u^{*} \mu_{1}^{*}+\mu_{2}^{*}=0, \quad \frac{\partial L_{1}^{*}}{\partial v^{*}}=2 v^{*}-4+2 v^{*} \mu_{1}^{*}+\mu_{2}^{*}=0, \\
& \mu_{1}^{*}\left(u^{*^{2}}+v^{*^{2}}-9\right)=0, \quad \mu_{2}^{*}\left(u^{*}+v^{*}-5\right)=0 .
\end{aligned}
$$

When $\mu_{1}^{*}>0$ and $\mu_{2}^{*}=0$, we have

$$
\begin{aligned}
& 2 u^{*}-5+2 u^{*} \mu_{1}^{*}=0 \Rightarrow u^{*}=\frac{5}{2\left(1+\mu_{1}^{*}\right)}, \\
& 2 v^{*}-4+2 v^{*} \mu_{1}^{*}=0 \Rightarrow v^{*}=\frac{4}{2\left(1+\mu_{1}^{*}\right)} .
\end{aligned}
$$

Since $\mu_{1}^{*}>0 \Rightarrow u^{*^{2}}+v^{*^{2}}-9=0$. Then, $\mu_{1}^{*}=0.06718 \Rightarrow\left(u^{*}, V^{*}\right)=(2.3426,1.874)$.

When $\mu_{2}^{*}>0$ and $\mu_{1}^{*}=0$, we have

$$
\begin{aligned}
& 2 u^{*}-5+\mu_{2}^{*}=0 \Rightarrow u^{*}=\frac{5-\mu_{2}^{*}}{2}, \\
& 2 v^{*}-4+\mu_{2}^{*}=0 \Rightarrow v^{*}=\frac{4-\mu_{2}^{*}}{2} .
\end{aligned}
$$

Since $\mu_{2}^{*}>0 \Rightarrow u^{*}+v^{*}-5=0$. Then, $\mu_{2}^{*}=-12$. This solution is refused.

So, the upper optimal solution point $\left(u^{*}, V^{*}\right)=(2.3426,1.874)$ is a surely optimal solution point then the stability of the $1^{\text {st }}$ kind will be obtained as follows:

$$
2(1.874)+P_{2}+2(1.874) \mu_{1}^{*}=0 \quad \Rightarrow \quad \mu_{1}^{*}=\frac{-3.748-P_{2}}{3.748} .
$$

Since $\mu_{1}^{*}>0 \Rightarrow-3.748-P_{2}>0 \Rightarrow P_{2}<-3.748$.

So the stability of the $1^{\text {st }}$ kind corresponds to the case

$$
\left(P_{2}<-3.748\right) \text {. }
$$

\section{Conclusion}

This paper presented a parametric study of the rough continuous static game when parameters exist in the crisp cost function and constraints are rough sets. Furthermore, the paper provided new concepts including the solvability set and the stability set of the $1^{\text {st }}$ kind and the possibly stability set in a rough environment. Also, the surely and possibly optimal solution sets of RCSG in the $1^{\text {st }}$ class are introduced. Future work may focus on the parametric study of the PRCSG when parameters are found in the constraints and the cost function is a rough function. 


\section{REFERENCES}

1. Bank B., Guddat J., Klatte D., Kummer B., Tammer K. Non-Linear Parametric Optimization. Basel: Birkhäuser, 1982. 228 p. DOI: 10.1007/978-3-0348-6328-5

2. Bazaraa M.S., Sherali H. D., Shetty C.M. Nonlinear Programming: Theory and Algorithms. 3rd Ed. Verlag: J. Wiley \& Sons Inc., 2013. 872 p.

3. Bertsekas P. D. Nonlinear Programming. 2rd Ed. Belmont, Massachusetts: Athena Scientific, 1999.791 p.

4. Budhiraja A., Dupuis P. Representations for functional of Hilbert space valued diffusions. In: Stochastic Analysis, Control, Optimization and Applications. McEneaney W.M., Yin G.G., Zhang Q. (eds.) Ser. Systems Control Found. Appl. Boston, MA: Birkhäuser, 1999. P. 1-20. DOI: $10.1007 / 978-1-4612-1784-8 \_1$

5. Elsisy M. A., Eid M. H., Osman M. S. A. Qualitative analysis of basic notions in parametric rough convex programming (parameters in the objective function and feasible region is a rough set). OPSEARCH, 2017. Vol. 54. P. 724-734. DOI: 10.1007/s12597-017-0300-2

6. Jongen H. Th., Jonker P., Twilt F. Nonlinear Optimization in Finite Dimensions. Boston, MA: Springer, 2000. 513 p. DOI: $10.1007 / 978-1-4615-0017-9$

7. Kalaiselvi R., Kousalya K. Statistical modelling and parametric optimization in document fragmentation. Neural Comput. Applic., 2020. Vol. 32. P. 5909-5918. DOI: 10.1007/s00521-019-04068-1

8. Lijun X., Yijia Z., Bo Y. Robust Optimization Model with Shared Uncertain Parameters in Multi-Stage Logistics Production and Inventory Process. Mathematics, 2020. Vol. 8, No. 2. Art. no. 211. P. 1-12. DOI: $10.3390 /$ math8020211

9. Matsumoto A., Szidarovszky F. Continuous Static Games. In: Game Theory and Its Applications. Tokyo: Springer, 2016. P. 21-47. DOI: 10.1007/978-4-431-54786-0_3

10. Miettinen K. Nonlinear Multiobjective Optimization. Ser. Internat. Ser. Oper. Res. Management Sci., vol. 12. NY: Springer, 1998. 298 p. DOI: 10.1007/978-1-4615-5563-6

11. Nguyen V., Gupta S., Rana S. et al. Filtering Bayesian optimization approach in weakly specified search space. Knowl. Inf. Syst., 2019. Vol. 60. P. 385-413. DOI: 10.1007/s10115-018-1238-2

12. Osman M. S. A. Qualitative analysis of basic notions in parametric convex programming. I. Parameters in the constraints. Aplikace Matematiky, 1977. Vol. 22., No. 5. P. 318-332. DOI: 10.21136/AM.1977.103710

13. Osman M., Lashein E. F., Youness E. A., Elsayed T. Mathematical programming in rough environment. Optimization, 2011. Vol. 60, No. 5. P. 603-611. DOI: 10.1080/02331930903536393

14. Patil A., Desai A. D. Parametric optimization of engine performance and emission for various $n$-butanol blends at different operating parameter condition. Alexandria Eng. J., 2020. Vol. 59, No. 2. P. 851-864. DOI: $10.1016 /$ j.aej.2020.02.006

15. Sawaragi Y., Nakayama H., Tanino T. Theory of Multiobjective Optimization. Math. Sci. Eng., vol. 176. Academic Press, 1985. 322 p.

16. Schneider J. J., Kirkpatrick S. Stochastic Optimization. Berlin Heidelberg: Springer-Verlag, 2006.568 p. DOI: $10.1007 / 978-3-540-34560-2$

17. Sun W., Yuan Y.-X. Optimization Theory and Methods: Nonlinear Programming. Springer Optim. Appl., vol. 1. US: Springer-Verlag, 2006. 688 p. DOI: 10.1007/b106451

18. Tuy H. Minimax: existence and stability. In: Pareto Optimality, Game Theory and Equilibria. A. Chinchuluun, P.M. Pardalos, A. Migdalas, L. Pitsoulis (eds.). Springer Optim. Appl., vol 17. NY: Springer. P. 3-21. DOI: 10.1007/978-0-387-77247-9_1

19. Youness E. Characterizing solutions of rough programming problems. European J. Oper. Res., 2006. Vol. 168, No. 3. P. 1019-1029. DOI: 10.1016/j.ejor.2004.05.019

20. Zhang J., Liu N., Wang S. A parametric approach for performance optimization of residential building design in Beijing. Build. Simul., 2019. Vol. 13. P. 223-235. DOI: 10.1007/s12273-019-0571-z 